\title{
Fragmented QRS as a Marker of Electrical Dyssynchrony to Predict Inter-Ventricular Conduction Defect by Subsequent Echocardiographic Assessment in Symptomatic Patients of Non-Ischemic Dilated Cardiomyopathy
}

\author{
Santosh Kumar Sinha ${ }^{a}$, b Kush Bhagat ${ }^{a}$, Mohammad Asifa, Karandeep Singh ${ }^{a}$, Mohit Sachan ${ }^{a}$, Vikas Mishra ${ }^{a}$, \\ Nasar Afdaalia, Mukesh Jitendra Jha ${ }^{\mathrm{a}}$, Ashutosh Kumar ${ }^{\mathrm{a}}$, Shravan Singh ${ }^{\mathrm{a}}$, Rupesh Sinha ${ }^{\mathrm{a}}$, \\ Dibbendhu Khanra $^{\text {a }}$, Ramesh Thakur ${ }^{\mathrm{a}}$, Chandra Mohan Varma ${ }^{\mathrm{a}}$, Vinay Krishna ${ }^{\mathrm{a}}$, Umeshwar Pandey ${ }^{\mathrm{a}}$
}

\begin{abstract}
Background: Left ventricular (LV) dyssynchrony frequently occurs in patients with heart failure (HF). QRS $\geq 120 \mathrm{~ms}$ is a surrogate marker of electrical dyssynchrony, which occurs in only $30 \%$ of HF patients. In contrary, in those with normal QRS (nQRS) duration, LV dyssynchrony has been reported in $20-50 \%$. This study was carried out to investigate the role of fragmented QRS (fQRS) on the surface electrocardiography (ECG) as a marker of electrical dyssynchrony to predict the presence of significant intraventricular dyssynchrony (IVD) by subsequent echocardiographic assessment.
\end{abstract}

Methods: A total of 226 consecutive patients with non-ischemic cardiomyopathy were assessed for fQRS on surface ECG as defined by presence of an additional $R$ wave ( $R$ prime), notching in nadir of the $S$ wave, notching of $\mathrm{R}$ wave, or the presence of more than one $\mathrm{R}$ prime (fragmentation) in two contiguous leads corresponding to a major myocardial segment. Tissue Doppler imaging (TDI) was performed in the apical views (four-chamber, two-chamber and long-axis) to analyze all 12 segments at both basal and middle levels. Time-topeak myocardial sustained systolic (Ts) velocities were calculated. Significant systolic IVD was defined as Ts-SD $>32.6 \mathrm{~ms}$ as known as "Yu index".

Result: Of the total patients, 112 had fQRS (49.5\%), while 114 had nQRS $(50.5 \%)$ with male dominance $(\mathrm{M} / \mathrm{F}=71: 29)$. Majority of patients were in NYHA class II $(\mathrm{n}=122,54 \%)$ followed by class III $(\mathrm{n}=$ $83 ; 37 \%)$, and class IV $(\mathrm{n}=21 ; 9 \%)$. There were no significant differences among both groups for baseline parameters except higher QRS duration (102.42 \pm 14.05 vs. $91.10 \pm 13.75 \mathrm{~ms} ; \mathrm{P}=0.001)$, higher Yu index (35.64 \pm 12.79 vs. $20.45 \pm 11.17 ; \mathrm{P}=0.01)$ and number of patients with positive $\mathrm{Yu}$ index $(78.6 \%$ vs. $21.1 \%$; $\mathrm{P}=0.04)$ in

Manuscript accepted for publication August 24, 2016

aDepartment of Cardiology, LPS Institute of Cardiology, G.S.V.M. Medical College, Kanpur, Uttar Pradesh 208002, India

${ }^{b}$ Corresponding Author: Santosh Kumar Sinha, Department of Cardiology, LPS Institute of Cardiology, G.S.V.M. Medical College, Kanpur, Uttar Pradesh 208002, India. Email: fionasan@rediffmail.com

doi: http://dx.doi.org/10.14740/cr495w group with fQRS compared with group with nQRS. fQRS complexes had $84.61 \%$ sensitivity and $80.32 \%$ specificity with positive predictive value of $78.6 \%$ and negative predictive value of $85.9 \%$ to detect IVD. On detailed segmental analysis for fQRS distribution, inferior segment had maximum (37\%), followed by anterior $(23 \%)$, lateral $(19 \%)$, inferior and lateral $(11 \%)$, anterior and inferior $(8 \%)$, and anterior and lateral (2\%). Among 104 patients with significant dyssynchrony, 88 patients $(84.6 \%)$ had fQRS in the dyssynchronic segment.

Conclusion: Fragmentation of QRS complex is an important predictor of electro-mechanical dyssynchrony. It is also helpful in localizing the dyssynchronous segment. In future, larger studies may be carried out to investigate the role of $\mathrm{fQRS}$ as a predictor of response to cardiac resynchronization therapy (CRT) in this subgroup of HF patients with narrow QRS.

Keywords: Fragmented QRS; Electrical dyssynchrony; Inter-ventricular conduction defect; Yu index; Tissue Doppler imaging

\section{Introduction}

Heart failure (HF) is a cardiovascular disease with increasing incidence and prevalence with high mortality rate and is the most common cause of hospitalization in patients with cardiac diseases. Left ventricular (LV) dyssynchrony frequently occurs in HF patients, especially in those with wide QRS interval [1]. Recently, management of HF has gone a paradigm shift from drugs to device in form of cardiac resynchronization therapy (CRT) among patients with wide QRS intervals which does optimization of the AV-delay resulting in synchrony in ventricular contraction and an improvement in systolic function and mitral regurgitation (MR) [2]. Despite these impressive results, almost $30-50 \%$ of patients are suboptimal responders in terms of clinical symptoms, and LV function assessed on echocardiography [3-5]. Reasons may be insufficient evidence of mechanical dyssynchrony pre-implantation, presence of transmural scar at posterolateral wall, lack of contractile reserve, severe MR, suboptimal LV lead position and inappropriate device programming [6]. LV mechanical dyssynchrony is 


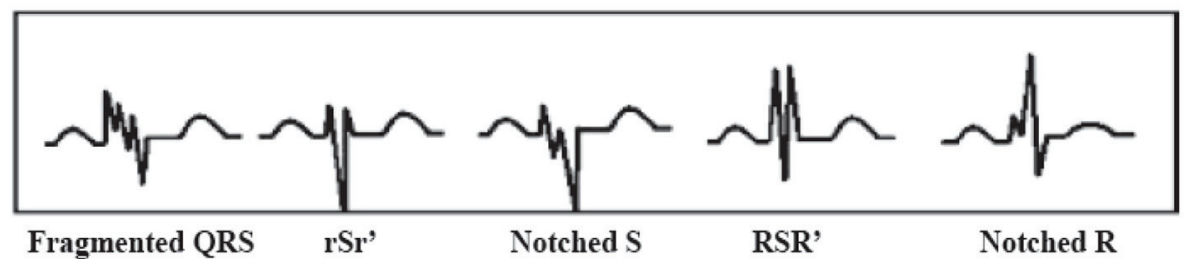

Figure 1. Different types of fragmented QRS (fQRS).

characterized by temporal differences in contraction between various myocardial segments, and commonly observed in patients with congestive HF, whose presence depends on assessment methodology, characteristics of the study population including the QRS duration, loading condition, severity of coronary artery disease, LV hypertrophy and LV remodeling. Therefore, the ECG criteria of QRS width $\geq 120 \mathrm{~ms}$ adopted in the current guidelines may be a suboptimal tool in identifying the patients who benefit most from CRT or defining the presence of mechanical dyssynchrony. The myocardial scar causes heterogeneous ventricular activity, and is associated with alteration in QRS morphology, leading to terminal conduction delay or fragmentation of the QRS complexes on 12lead ECG [7]. Fragmented QRS (fQRS) includes various RSR patterns with different morphologies of the QRS complexes with or without the Q wave on a resting 12-lead ECG [8]. It is associated with subendocardial fibrosis and myocardial scars and it has been shown that response to CRT is associated with the extent of myocardial scar tissue in patients with ventricular dyssynchrony [9-11]. Because of large inter-individual variability to response to CRT, it is important to find the predictor's responsiveness pre-implantation. QRS duration is the main selection criterion for diagnosing the presence of mechanical dyssynchrony. However, it is poorly correlated with the presence of mechanical dyssynchrony as measurement of QRS duration is an electrical phenomenon. Though $\mathrm{QRS} \geq 120 \mathrm{~ms}$ is a surrogate marker of electrical dyssynchrony, it occurs in only $30 \%$ of HF patients. On the other hand, in patients of HF with normal QRS duration, LV dyssynchrony has been reported in $20-50 \%[12,13]$. fQRS in patients with non-ischemic dilated cardiomyopathy (DCM) is related to uncoordinated depolarization of viable myocytes being surrounded by fibrotic tissue as a result of inflammation of myocytes causing necrosis and fibrosis which eventually leads to mechanical dyssynchrony $[14,15]$. This study was carried out to investigate the role of fQRS on the surface ECG as a marker of electrical dyssyn-

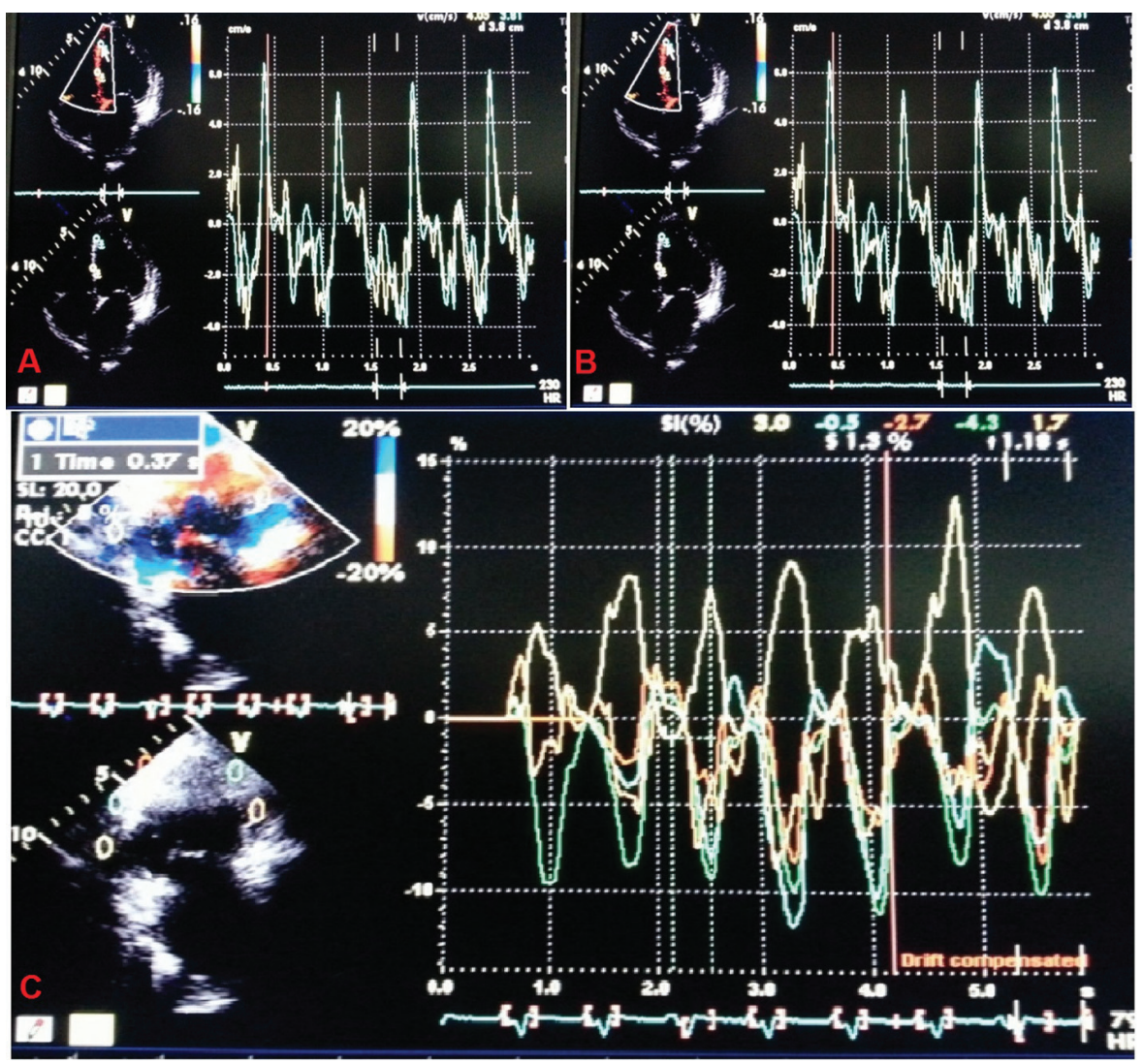

Figure 2. Tissue Doppler imaging to calculate "Yu index". 
Table 1. Baseline Clinical, Electrocardiographic and Echocardiographic Parameters of Patients $(n=226)$

\begin{tabular}{llll}
\hline Variables & fQRS $(\mathbf{n}=\mathbf{1 1 2} ; \mathbf{\%})$ & nQRS $(\mathbf{n}=\mathbf{1 1 4} ; \mathbf{\%})$ & P value \\
\hline Age (years) & $45.88 \pm 13.70$ & $47.84 \pm 14.07$ & 0.48 \\
\hline Sex (M/F) & $81(73): 31(27)$ & $79(69): 35(31)$ & 0.56 \\
NYHA class (II/III/IV) & $59(52) / 40(35) / 13(11)$ & $63(55) / 43(38) / 8(7)$ & 0.22 \\
\hline Medications use & & & \\
$\quad$ Beta blocker & $96(86)$ & $102(89.5)$ & 0.53 \\
$\quad 105(94)$ & $110(96.5)$ & 0.53 \\
$\quad$ Diuretic & $85(76)$ & $93(81.5)$ & 0.66 \\
$\quad$ ACE-I/ ARB & $43(38.4)$ & $49(43)$ & 0.66 \\
$\quad 102.42 \pm 14.05$ & $91.10 \pm 13.75$ & 0.001 \\
QRS duration (ms) & $28.24 \pm 5.08$ & $27.245 \pm 9.2$ & 0.07 \\
LVEF (\%) & $62.42 \pm 8.10$ & $60.6 \pm 8.01$ & 0.28 \\
LVEDD (mm) & $54.1 \pm 8.5$ & $51.8 \pm 7.32$ & 0.13 \\
LVESD (mm) & $35.64 \pm 12.79$ & $20.45 \pm 11.17$ & 0.01 \\
Ts-SD (Yu index) & $88(78.6)$ & $24(21.1)$ & 0.04 \\
\hline Yu index positive patient & & & \\
\hline
\end{tabular}

NYHA: New York Heart Association; ACE-I: angiotensin converting enzyme inhibitor; ARB: angiotensin receptor blocker; LVEF: left ventricular ejection fraction; LVEDD: left ventricular end-diastolic dimension; LVESD: left ventricular endsystolic dimension; fQRS: fragmented QRS; nQRS: normal QRS.

chrony to predict the presence of significant intraventricular dyssynchrony (IVD) by subsequent echocardiographic assessment.

\section{Materials and Methods}

It was a prospective, non-randomized, single-center study of 226 patients conducted in the Department of Cardiology, LPS Institute of Cardiology, G.S.V.M. Medical College, Kanpur, UP, India, from September 2013 till the end of July 2015. Inclusion criteria were all symptomatic patients with chronic HF (NYHA functional class $\geq$ II) with guideline-directed medical treatment for at least 6 months due to non-ischemic DCM, LV ejection fraction (LVEF) $\leq 35 \%$ and normal sinus rhythm having narrow QRS complexes $(\leq 120 \mathrm{~ms})$. Exclusion criteria were patients with valvular heart disease, history of myocardial infarction, ischemic ECG findings, angiographically significant coronary artery disease $(\geq 70 \%$ stenosis in any epicardial coronary artery), atrial fibrillation, chronic liver or kidney failure, as well as patients with permanent pacemakers.

All patients were comprehensively evaluated including their 12-lead ECG. Their functional capacity was recorded. Coronary angiogram was done in all patients to rule out the presence of significant coronary artery disease.

\section{ECG}

fQRS included various RSR patterns and was defined by the presence of an additional $\mathrm{R}$ wave ( $\mathrm{R}$ prime), notching in nadir of the $\mathrm{S}$ wave, notching of $\mathrm{R}$ wave, or the presence of more than one $\mathrm{R}$ prime (fragmentation) in two contiguous leads corresponding to a major myocardial segment as previously described by Das et al [8] (Fig. 1). The presence of fQRS in $\geq 2$ contiguous anterior leads $\left(\mathrm{V}_{1}-\mathrm{V}_{5}\right)$ were assigned to anterior myocardial segments, $\geq 2$ contiguous lateral leads (I, aVL, and $\mathrm{V}_{5}, \mathrm{~V}_{6}$ ) to the lateral myocardial segments, $\geq 2$ contiguous inferior leads (II, III, and aVF) to the inferior myocardial segments and both $\mathrm{V}_{1}$ and $\mathrm{V}_{2}$ only to the posterior myocardial segments.

\section{Echocardiography}

Echocardiography was performed with VIVID7 (GE) instrument. Tissue Doppler imaging (TDI) was performed in the apical views (four-chamber, two-chamber and long-axis) for the long axis motion of the left ventricle. Myocardial regional velocity curves were constructed from the digitized images using Q analysis by dedicated offline software. In this way, all 12 segments were analyzed at both basal and middle levels. Measurement was taken at the beginning of the QRS complex considering this as the reference point, from where the timeto-peak myocardial sustained systolic (Ts) velocities were calculated. For the assessment of synchronicity, the standard deviations of Ts (Ts-SD) of all 12 LV segments were calculated. Significant systolic IVD was defined as Ts-SD > $32.6 \mathrm{~ms}$ as proposed by Yu et al [16] and popularly known as "Yu index" (Fig. 2). "Dyssynchronic segments" were defined as those LV segments that contracted later than $100 \mathrm{~ms}$ following the earliest contracting LV segment (which had the earliest Ts) and "most delayed segment" was the last contracting myocardial segment (which had the latest Ts). The study protocol was approved by the local ethics committee and followed the Declaration of Helsinki after obtaining informed consent from each 


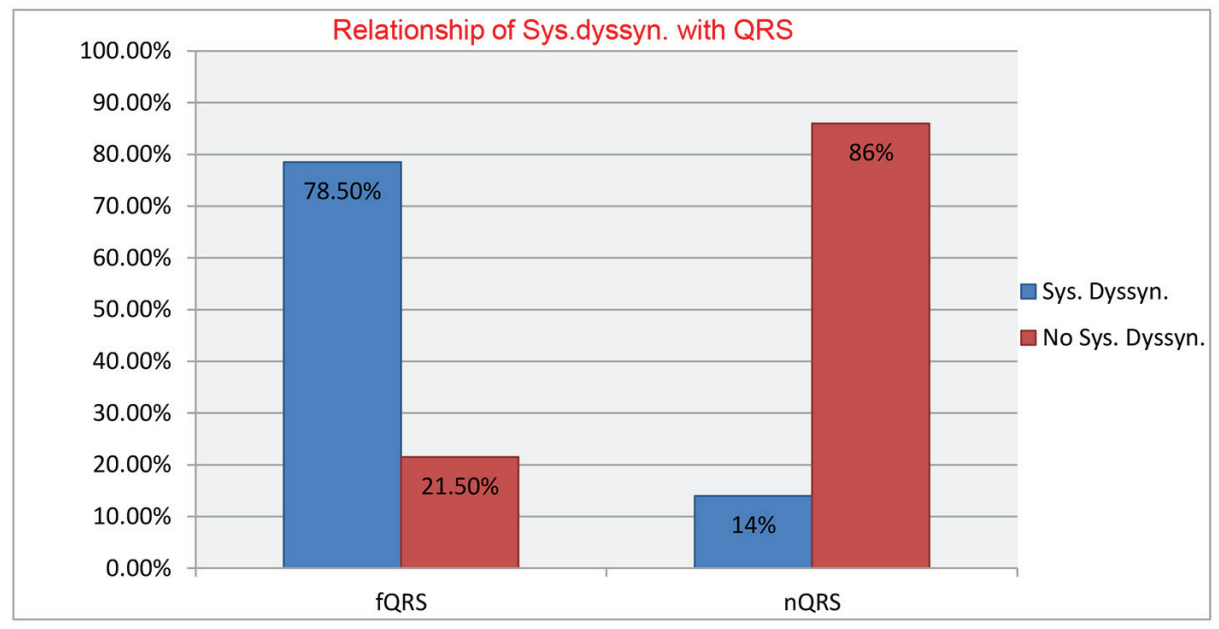

Figure 3. Sensitivity, specificity, positive predictive value and negative predictive value of fQRS complexes to detect intraventricular dyssynchrony.

patient.

\section{Statistical analysis}

Statistical analyses were performed using the SPSS 17.0 (SPSS Inc, Chicago, IL). Continuous variables were analyzed using Student's $t$-test and presented as mean $\pm \mathrm{SD}$, whereas categorical variables were given as numbers (percentages). The comparison between groups was done by Mann-Whitney $\mathrm{U}$ test for continuous variables and by Chi-square or Fisher's exact test for categorical variables. $\mathrm{P}<0.05$ was considered statistically significant.

\section{Results}

Two hundred and twenty-six patients of non-ischemic cardiomyopathy were investigated, of which 112 had fQRS, while 114 patients had nQRS whose baseline characteristics are shown in Table 1 . There was male dominance $(\mathrm{M} / \mathrm{F}=71: 29)$ with no significant difference among both groups. Majority of patients were in NYHA class II $(122,54 \%)$, followed by class III $(\mathrm{n}=83 ; 37 \%)$, and class IV $(\mathrm{n}=21 ; 9 \%)$. There were no significant differences among both groups for baseline parameters except higher QRS duration (102.42 \pm 14.05 vs. $91.10 \pm$ $13.75 \mathrm{~ms} ; \mathrm{P}=0.001)$, higher Yu index (35.64 \pm 12.79 vs. 20.45 $\pm 11.17 ; \mathrm{P}=0.01)$ and number of patients with positive $\mathrm{Yu}$ index $(78.6 \%$ vs. $21.1 \% ; \mathrm{P}=0.04)$ in group with fQRS compared with group with nQRS. fQRS complexes had $84.61 \%$ sensitivity and $80.32 \%$ specificity with positive predictive value of $78.6 \%$ and negative predictive value of $85.9 \%$ to detect IVD (Fig. 3). When detailed segmental analysis was performed for the distribution of fQRS, inferior segment had maximum (37\%) followed by anterior (23\%), lateral (19\%), inferior and lateral $(11 \%)$, anterior and inferior $(8 \%)$, and anterior and lateral (2\%) (Fig. 4). Among 104 patients with significant dyssynchrony, 88 patients $(84.6 \%)$ had ECG fragmentation in the dyssynchronic segment.

\section{Discussion}

The predominant mechanism of benefit from CRT appears to be related to the presence of electro-mechanical dyssynchrony, i.e. $\mathrm{QRS} \geq 120 \mathrm{~ms}$ and subsequent resynchronization after CRT [17-20]. However, studies have shown that QRS duration of $\geq 120 \mathrm{~ms}$ occurs in only $30 \%$ of HF, and significant IVD by TDI has been reported in $36-51 \%$ of patients with HF and QRS $\leq 120 \mathrm{~ms}$, and these patients also showed benefit from CRT [21-24]. There are few trials showing benefit of CRT with NYHA class III/IV HF and EF $\leq 35 \%$, but QRS $\leq 120 \mathrm{~ms}$, who had mechanical dyssynchrony defined as a septal-to-lateral wall time-to-peak systolic velocity delay of $\geq$ $65 \mathrm{~ms}$ by TDI as shown by Bleeker et al [25]. With QRS prolongation, shape of QRS complex also changes, with LBBB being the most common followed by RBBB. fQRS is present in $23-75 \%$ of the patients with idiopathic DCM and narrow QRS complexes.

Das et al [8] first reported the significance of fQRS in patients of coronary artery disease where it was found to be a better predictor of myocardial scar than Q waves on ECG. In non-ischemic cardiomyopathy, it is electrical manifestation of uncoordinated depolarization of viable myocytes surrounded by fibrotic tissue, which eventually leads to mechanical dyssynchrony.

Tigen et al [1] were the first to investigate the relationship between fQRS and IVD in patients of non-ischemic DCM and narrow QRS interval (duration $\leq 120 \mathrm{~ms}$ ). They defined significant systolic dyssynchrony as the maximum difference in peak systolic velocities between any two LV segments of more than $100 \mathrm{~ms}$. Using this criterion, fQRS was found to detect IVD in $72.5 \%$ patients with sensitivity of $90.6 \%$ and positive predictive value of $72.5 \%$. Subsequently, Basaran et al [9] reported that $\mathrm{fQRS}$ on the surface ECG in patients of non-ischemic DCM is associated with significant IVD in $76 \%$ 


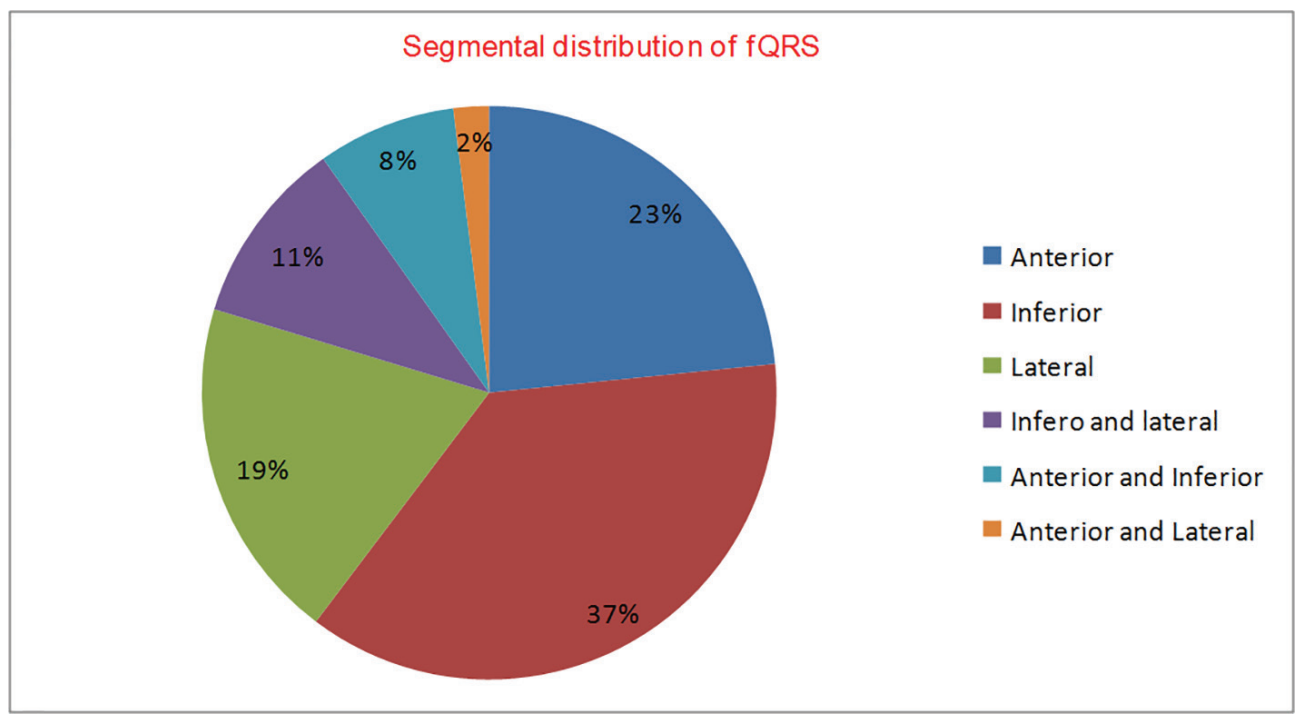

Figure 4. Segmental distribution of fQRS $(n=114)$.

patients as assessed by the "Yu index". But due to lack of control group of patients without fQRS, they could not assess the sensitivity or positive predictive value of $\mathrm{fQRS}$ as predictor of significant IVD.

"Yu index" has been shown to be an excellent predictor of CRT responder defined by reverse remodeling ( $\geq 15 \%$ reduction in LV end-systolic volume). In patients with QRS duration $\geq 150 \mathrm{~ms}$, the "Yu index" has a sensitivity and specificity of $100 \%$ and $78 \%$, respectively to predict reverse remodeling, while in patients with QRS duration of 120 - $150 \mathrm{~ms}$, the sensitivity is $83 \%$ and specificity is $86 \%$ [15]. "Yu index" is based on 12 segment models using color-coded TDI integrating information from three apical views (four-chamber, two-chamber and apical long axis view) in contrast to the simple two segment (apical four-chamber view to detect basal septal-lateral delay) and four segment (basal segments of septal, lateral, inferior and anterior walls) approaches to detect IVD. This model is more comprehensive as it incorporates the anterior septum and posterior walls which often displays dyssynchrony. Our study showed that fQRS had a sensitivity of $84.61 \%$ and a positive predictive value of $78.6 \%$ to detect significant IVD as defined by the "Yu index".

Beshai et al [26] in RethinQ trial investigated the role of CRT in HF patients with narrow QRS and echocardiographic evidence of IVD. They showed that IVD per se failed to provide clinical benefits with CRT over medial therapy in the absence of ECG evidence of electrical dyssynchrony. Our study has shown that fQRS on the surface ECG is a marker of electrical dyssynchrony and can predict significant IVD as defined by the "Yu index" in more than $80 \%$ patients. In our study, QRS duration was significantly greater in patients with fQRS as compared to those with nQRS, although both groups had QRS duration of $\leq 120 \mathrm{~ms}$. As fragmentation along with widening of QRS leads to electrical dyssynchrony, fQRS in patients with QRS $\leq 120 \mathrm{~ms}$ may be considered to have the same significance as wide QRS to predict the presence of electrical dyssynchrony leading to IVD and responsiveness to CRT in patients of non-ischemic DCM.

\section{Conclusion}

Fragmentation of QRS complex is an important predictor of electrical dyssynchrony. It is also helpful in localizing the dyssynchronous segment. In future, larger studies may be carried out to investigate the role of $\mathrm{fQRS}$ as a predictor of response to CRT in this subgroup of HF patients with narrow QRS.

\section{Financial Disclosure}

None.

\section{Conflicts of Interest}

None.

\section{References}

1. Tigen K, Karaahmet T, Gurel E, Cevik C, Nugent K, Pala $\mathrm{S}$, Tanalp AC, et al. The utility of fragmented QRS complexes to predict significant intraventricular dyssynchrony in nonischemic dilated cardiomyopathy patients with a narrow QRS interval. Can J Cardiol. 2009;25(9):517-522.

2. Versteeg H, Schiffer AA, Widdershoven JW, Meine MM, Doevendans PA, Pedersen SS. Response to cardiac resynchronization therapy: is it time to expand the criteria? Pacing Clin Electrophysiol. 2009;32(10):1247-1256.

3. Lecoq G, Leclercq C, Leray E, Crocq C, Alonso C, de Place C, Mabo P, et al. Clinical and electrocardiographic predictors of a positive response to cardiac resynchronization therapy in advanced heart failure. Eur Heart J. 
2005;26(11):1094-1100.

4. Mollema SA, Bleeker GB, van der Wall EE, Schalij MJ, Bax JJ. Usefulness of QRS duration to predict response to cardiac resynchronization therapy in patients with endstage heart failure. Am J Cardiol. 2007;100(11):16651670.

5. Buck S, Maass AH, Nieuwland W, Anthonio RL, Van Veldhuisen DJ, Van Gelder IC. Impact of interventricular lead distance and the decrease in septal-to-lateral delay on response to cardiac resynchronization therapy. Europace. 2008;10(11):1313-1319.

6. Hekmatpou D, Mohammadi E, Ahmadi F, Arefi SH. Noncompliance factors of congestive heart failure patients readmitted in cardiac care units. IJCCN. 2009;23:91-97.

7. Aslani A, Tavoosi A, Emkanjoo Z. Diffuse fragmented QRS as an index of extensive myocardial scar. Indian Pacing Electrophysiol J. 2010;10(1):67-68.

8. Das MK, Suradi H, Maskoun W, Michael MA, Shen C, Peng J, Dandamudi G, et al. Fragmented wide QRS on a 12-lead ECG: a sign of myocardial scar and poor prognosis. Circ Arrhythm Electrophysiol. 2008;1(4):258-268.

9. Basaran Y, Tigen K, Karaahmet T, Isiklar I, Cevik C, Gurel E, Dundar C, et al. Fragmented QRS complexes are associated with cardiac fibrosis and significant intraventricular systolic dyssynchrony in nonischemic dilated cardiomyopathy patients with a narrow QRS interval. Echocardiography. 2011;28(1):62-68.

10. Adelstein EC, Saba S. Scar burden by myocardial perfusion imaging predicts echocardiographic response to cardiac resynchronization therapy in ischemic cardiomyopathy. Am Heart J. 2007;153(1):105-112.

11. Ypenburg C, Schalij MJ, Bleeker GB, Steendijk P, Boersma E, Dibbets-Schneider P, Stokkel MP, et al. Impact of viability and scar tissue on response to cardiac resynchronization therapy in ischaemic heart failure patients. Eur Heart J. 2007;28(1):33-41.

12. Kashani A, Barold SS. Significance of QRS complex duration in patients with heart failure. J Am Coll Cardiol. 2005;46(12):2183-2192.

13. Freudenberger R, Sikora JA, Fisher M, Wilson A, Gold M. Electrocardiogram and clinical characteristics of patients referred for cardiac transplantation: implications for pacing in heart failure. Clin Cardiol. 2004;27(3):151153.

14. Marijianowski MM, Teeling P, Mann J, Becker AE. Dilated cardiomyopathy is associated with an increase in the type I/type III collagen ratio: a quantitative assessment. J Am Coll Cardiol. 1995;25(6):1263-1272.

15. Yu CM, Fung JW, Chan CK, Chan YS, Zhang Q, Lin H, Yip GW, et al. Comparison of efficacy of reverse remodeling and clinical improvement for relatively narrow and wide QRS complexes after cardiac resynchronization therapy for heart failure. J Cardiovasc Electrophysiol. 2004;15(9):1058-1065.
16. Yu CM, Chan YS, Zhang Q, Yip GW, Chan CK, Kum LC, $\mathrm{Wu} \mathrm{L}$, et al. Benefits of cardiac resynchronization therapy for heart failure patients with narrow QRS complexes and coexisting systolic asynchrony by echocardiography. J Am Coll Cardiol. 2006;48(11):2251-2257.

17. Bax JJ, Marwick TH, Molhoek SG, Bleeker GB, van Erven L, Boersma E, Steendijk P, et al. Left ventricular dyssynchrony predicts benefit of cardiac resynchronization therapy in patients with end-stage heart failure before pacemaker implantation. Am J Cardiol. 2003;92(10):1238-1240.

18. Yu CM, Fung WH, Lin H, Zhang Q, Sanderson JE, Lau CP. Predictors of left ventricular reverse remodeling after cardiac resynchronization therapy for heart failure secondary to idiopathic dilated or ischemic cardiomyopathy. Am J Cardiol. 2003;91(6):684-688.

19. Breithardt OA, Stellbrink C, Kramer AP, Sinha AM, Franke A, Salo R, Schiffgens B, et al. Echocardiographic quantification of left ventricular asynchrony predicts an acute hemodynamic benefit of cardiac resynchronization therapy. J Am Coll Cardiol. 2002;40(3):536-545.

20. Bax JJ, Bleeker GB, Marwick TH, Molhoek SG, Boersma E, Steendijk P, van der Wall EE, et al. Left ventricular dyssynchrony predicts response and prognosis after cardiac resynchronization therapy. J Am Coll Cardiol. 2004;44(9):1834-1840.

21. Cazeau S, Leclercq C, Lavergne T, Walker S, Varma C, Linde C, Garrigue S, et al. Effects of multisite biventricular pacing in patients with heart failure and intraventricular conduction delay. N Engl J Med. 2001;344(12):873880 .

22. Haghjoo M, Bagherzadeh A, Fazelifar AF, Haghighi ZO, Esmaielzadeh M, Alizadeh A, Emkanjoo Z, et al. Prevalence of mechanical dyssynchrony in heart failure patients with different QRS durations. Pacing Clin Electrophysiol. 2007;30(5):616-622.

23. Bleeker GB, Holman ER, Steendijk P, Boersma E, van der Wall EE, Schalij MJ, Bax JJ. Cardiac resynchronization therapy in patients with a narrow QRS complex. J Am Coll Cardiol. 2006;48(11):2243-2250.

24. Achilli A, Sassara M, Ficili S, Pontillo D, Achilli P, Alessi C, De Spirito S, et al. Long-term effectiveness of cardiac resynchronization therapy in patients with refractory heart failure and "narrow" QRS. J Am Coll Cardiol. 2003;42(12):2117-2124.

25. Bleeker GB, Schalij MJ, Molhoek SG, Holman ER, Verwey HF, Steendijk P, van der Wall EE, et al. Frequency of left ventricular dyssynchrony in patients with heart failure and a narrow QRS complex. Am J Cardiol. 2005;95(1):140-142.

26. Beshai JF, Grimm RA, Nagueh SF, Baker JH, 2nd, Beau SL, Greenberg SM, Pires LA, et al. Cardiac-resynchronization therapy in heart failure with narrow QRS complexes. N Engl J Med. 2007;357(24):2461-2471. 